Pacific

Journal of

Mathematics

SOME REMARKS ON VARIETIES WITH DEGENERATE GAUSS IMAGE

Emilia Mezzetti And Orsola Tommasi

Volume 213 No. 1

January 2004 


\title{
SOME REMARKS ON VARIETIES WITH DEGENERATE GAUSS IMAGE
}

\author{
Emilia Mezzetti And Orsola Tommasi
}

\begin{abstract}
We consider projective varieties with degenerate Gauss image whose focal hypersurfaces are non-reduced schemes. Examples of this situation are provided by the secant varieties of Severi and Scorza varieties. The Severi varieties are moreover characterized by a uniqueness property.
\end{abstract}

\section{Introduction.}

A classical theorem on surfaces states that, if the tangent plane to a ruled projective surface $S$ remains fixed along a general line of the ruling, then $S$ is a developable surface, i.e., a cone or the tangent developable of a curve.

In the case of higher dimensional varieties, this generalizes to the problem of giving a structure theorem for projective varieties with degenerate Gauss image. More precisely, let $X \subset \mathbb{P}^{N}$ be a projective variety of dimension $n$. The Gauss map of $X$ is the rational map $\gamma$ from $X$ to the Grassmannian $\mathbb{G}(n, N)$ of $n$-dimensional subspaces of $\mathbb{P}^{N}$, associating to a smooth point $x$ of $X$ the embedded tangent space $\mathbb{T}_{x} X$ to $X$ at $x$. The Gauss image $\gamma(X)$ of $X$ is, by definition, the closure of $\gamma\left(X_{\mathrm{sm}}\right)$, where $X_{\mathrm{sm}}$ is the smooth locus of $X$.

Clearly $\operatorname{dim} \gamma(X) \leq n$, and for "general" varieties equality holds. Several terms have been used recently to denote the varieties such that $\operatorname{dim} \gamma(X)<$ $\operatorname{dim} X$ : They are called varieties with degenerate Gauss image by Landsberg $([\mathbf{L 2}])$, developable by Piontkowski $([\mathbf{P}])$, tangentially degenerate by AkivisGoldberg ([AG]). We will follow Landsberg's convention.

Several general facts are well-known for these varieties (see for instance the classical article $[\mathbf{S}]$ ). First of all, general fibres of $\gamma$ are linear. This means that, if the dimension of the Gauss image is $r<n$, then the general fibre is a linear space of dimension $n-r$ along which the tangent space is constant. Secondly, if $X$ is not linear, then it is singular and its singular locus cuts a general fibre along a codimension 1 subscheme.

The theory of foci for families of linear varieties well applies to the family of fibres of $\gamma$. This allows us to consider and study the focal locus on $X$ and on the fibre $\Lambda$ of $\gamma$, which we will denote by $F$ and $F_{\Lambda}$ respectively. Both loci have a natural scheme structure. 
Several classification results have recently been established for these varieties (e.g., $[\mathbf{G H}],[\mathbf{A G L}],[\mathbf{M T}],[\mathbf{P}]$ and $[\mathbf{A G}]$ ) but a general structure theorem is still missing. In $[\mathbf{A G}]$ a structure theorem is proved, but under rather strong assumptions on a general $F_{\Lambda}$, for example that it is reduced or set-theoretically linear. Moreover, Akivis and Goldberg state the problem of constructing examples of projective varieties with degenerate Gauss image such that $F_{\Lambda}$ is not reduced and $\left(F_{\Lambda}\right)_{\text {red }}$ is not linear (or proving that such varieties do not exist).

Piontkowski $([\mathbf{P}])$ proved a uniqueness theorem for developable varieties $X$ with Gauss image of dimension 2, such that the focal locus has codimension 2 in $X$. Those for which the focal conic is integral are precisely the varieties of secant lines of the Veronese surface $v_{2}\left(\mathbb{P}^{2}\right)$ or of cones over it. Inspired by this example, we construct a series of examples where $F_{\Lambda}$ is not reduced and $\left(F_{\Lambda}\right)_{\text {red }}$ has arbitrarily large degree. The examples are the secant varieties of Severi varieties of dimension $>2$ and some natural generalizations of them.

Zak's theorem on linear normality $([\mathbf{Z 1}])$ states that a smooth non-degenerate $m$-dimensional subvariety of $\mathbb{P}^{N}$, with $N<3 m / 2+2$, cannot be isomorphically projected to $\mathbb{P}^{N-1}$. Zak also classified in $[\mathbf{Z 2}]$ the varieties which are borderline cases in his theorem and called them Severi varieties. He proved that there are only 4 smooth examples: The Veronese surface $v_{2}\left(\mathbb{P}^{2}\right)$ in $\mathbb{P}^{5}, \mathbb{P}^{2} \times \mathbb{P}^{2}, \mathbb{G}(1,5)$ and the non-classical variety $E$ of dimension 16. For any of these varieties $F$, its secant variety $X=S F$ is a cubic hypersurface in $\mathbb{P}^{N}$ with Gauss image isomorphic to $X$.

We prove in Example 2 that on a general fibre of the Gauss map for the secant variety of a Severi variety, the focal scheme is a quadric of maximal rank with multiplicity 1, 2, 4, 8 respectively.

In Section 4 we show that these examples can be generalized in two ways, taking instead of the Severi varieties the following three series of varieties $Y$ : $v_{2}\left(\mathbb{P}^{m}\right), \mathbb{P}^{m} \times \mathbb{P}^{m}$ and $\mathbb{G}(1,2 m+1)$, of dimensions $m, 2 m, 4 m$ respectively (they are Scorza varieties according to Zak $[\mathbf{Z 3}]$ ). If we consider their higher secant variety $S^{m-1} Y$ of $m$-secant $(m-1)$-planes, it turns out that they are hypersurfaces of degree $m+1$, whose dual is $Y$, and whose singular locus is $S^{m-2} Y$ and appears as focal locus in each fibre with multiplicity resp. 1, 2,4 . If we consider $S Y$ instead, we get on each fibre a focal quadric with an arbitrarily high multiplicity, more precisely, $m-1,2(m-1), 4(m-1)$ respectively.

It is interesting to observe that the fourth Severi variety does not generalize to give a complete class of examples $([\mathbf{L} \mathbf{1}])$.

We observe also that there is an upper bound on the codimension of the focal scheme and that there are restrictions on the set of possible codimensions if we require that $F$ is not reduced (see Theorem 3.1). For "high" codimension we prove in Theorem 3.3 some uniqueness results, in the case 
in which the general focal hypersurface is a quadric of maximal rank with multiplicity 2. We extend in this way the result found by Piontkowski in the case of the secant variety of the Veronese surface. Our results rely on the fact, stated by Zak in $[\mathbf{Z 4}]$, that if $F$ is a variety whose general linear section is a Severi variety, then $F$ is a cone over a Severi variety.

The plan of the article is as follows: In $\S 2$ we recall the language of focal schemes for families of linear spaces, as introduced in $[\mathbf{M T}]$. We also prove Proposition 2.2, giving a lower bound, based on the codimension of the focal locus, for the multiplicity of the focal scheme. In $\S 3$ we prove Theorems 3.1 and 3.3. Finally in $\S 4$, we give two series of examples, showing that the focal hypersurfaces can have arbitrarily high degree and multiplicity.

1.1. Notations and conventions. In this paper a variety will be an integral closed subscheme of a projective space over an algebraically closed field $\mathbb{K}$, char $\mathbb{K}=0$.

If $\Lambda \subset \mathbb{P}^{N}$ is a projective linear subspace, $\hat{\Lambda} \subset \mathbb{K}$ will denote the linear subspace associated to $\Lambda$ such that $\Lambda=\mathbb{P}(\hat{\Lambda})$. $T_{x} X$ will denote the Zariski tangent space to the variety $X$ at its point $x$, while we will denote by $\mathbb{T}_{x} X \subset$ $\mathbb{P}^{N}$ the embedded tangent space to $X$ at $x$.

We will always use the same symbol to denote the points of a Grassmannian and the corresponding linear subspaces.

\section{The focal scheme and its multiplicity.}

Let $X \subset \mathbb{P}^{N}$ be a projective variety of dimension $n<N$. Let us assume that $X$ is covered by an $r$-dimensional family of linear spaces of dimension $k:=$ $n-r$. Let $B$ be the subvariety of the Grassmannian $\mathbb{G}(k, N)$ parametrizing that family.

Let us denote by $\mathcal{I}$ the incidence correspondence of $B$, with the natural projections:

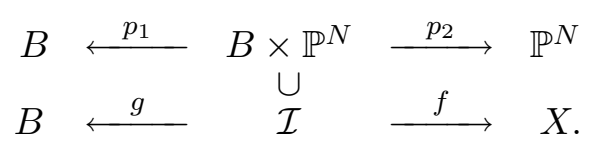

We will associate to the family $B$ its focal subscheme $\Phi \subset \mathcal{I}$ and its focal locus $F \subset X$. For their definitions and for the proof of results cited in this section, we refer to $[\mathbf{M T}]$.

We recall moreover that the characteristic map of the family $B$ is the map $\chi:=\beta \circ \alpha$,

$$
\left(p_{1}^{*}\left(\mathcal{T}_{B}\right)\right)_{\mid \mathcal{I}} \stackrel{\alpha}{\longrightarrow} \mathcal{T}_{\mathcal{I}} \stackrel{\beta}{\longrightarrow} \mathcal{N}_{\mathcal{I} \mid B \times \mathbb{P}^{N}}
$$

where $\alpha$ comes from the exact sequence expressing the tangent sheaf to the product variety $B \times \mathbb{P}^{N}$ as a product of tangent sheaves, and $\beta$ from that defining the normal sheaf to $\mathcal{I}$ inside $B \times \mathbb{P}^{N}$. 
For every smooth point $\Lambda$ of $B$, the restriction of $\chi$ to $g^{-1}(\Lambda)$ is called the characteristic map of $B$ relative to $\Lambda$. Finally the focal scheme on $\Lambda$, denoted $F_{\Lambda}$, is the scheme-theoretic intersection of the Cartier divisor $\Phi \subset \mathcal{I}$ with $\{\Lambda\} \times \Lambda \subset \mathcal{I}$.

Let $\gamma: X \rightarrow \mathbb{G}(n, N)$ be the (rational) Gauss map of $X$, regular on $X_{\mathrm{sm}}$. It is a consequence of biduality $([\mathbf{K}])$ that its fibres are linear spaces, i.e., that a general embedded tangent space is tangent to $X$ along a linear space. In the following, we will apply the above construction to the family of the fibres of $\gamma$, assuming that they are of positive dimension.

Definition 2.1. The Gauss rank of a variety $X$ is the number $r=\operatorname{dim} \gamma(X)$. If $r<n$, then $X$ is called a variety with degenerate Gauss image.

Varieties with degenerate Gauss image are characterized by the fact that the focal scheme on a general $\Lambda \in B$ is a hypersurface of degree $r$. Another important property is that the focal locus of $B$ is always contained in the singular locus of $X$.

Let us denote by $\bar{g}$ (respectively, $\bar{f}$ ) the restriction of $g$ (resp., $f$ ) to $\Phi \subset \mathcal{I}$. Note that the fibre $\bar{g}^{-1}(\Lambda)$ coincides with $F_{\Lambda}$, which has in general dimension $n-r-1$, hence every irreducible component of $\Phi$ has dimension $n-1$.

Proposition 2.2. Let $X$ be a variety of dimension $n$ with Gauss image of dimension $r<n$. Let $\bar{\Phi}$ be an irreducible component of the focal scheme of the family $B$ considered as a reduced variety. Let $\bar{F} \subset X$ be its schemetheoretic image and let $c$ be the codimension of $\bar{F}$ in $X$. For a general point $P=(\Lambda, x) \in \bar{\Phi}$, the Cartier divisor $\Phi \subset \mathcal{I}$ has Samuel multiplicity $\mu(P, \Phi) \geq c-1$.

Corollary 2.3. For a general element $(\Lambda, x) \in \bar{\Phi}$, either $F_{\Lambda}=\{\Lambda\} \times \Lambda$ or the Cartier divisor $F_{\Lambda} \subset\{\Lambda\} \times \Lambda$ has Samuel multiplicity $\mu\left(x, F_{\Lambda}\right) \geq c-1$.

Proof. Let $P=(\Lambda, x) \in \bar{\Phi}$ be a point that projects to $x$. Considering the differentials of the maps $f$ and $\bar{f}$ at $P$, we get the diagram:

$$
\begin{array}{ccc}
T_{P} \mathcal{I} & \stackrel{d_{P} f}{\longrightarrow} & T_{x} X \\
\cup & & \cup \\
T_{P} \bar{\Phi} & \stackrel{d_{P} \bar{f}}{\longrightarrow} & T_{x} \bar{F} .
\end{array}
$$

We consider also the characteristic map $\chi(\Lambda)$ of the family $B$ relative to $\Lambda$. At the point $P$, it gives rise to a linear map

$$
\chi(\Lambda, x): T_{\Lambda} B \rightarrow \hat{\mathbb{T}}_{x} X / \hat{\Lambda} .
$$

Let $K_{P}$ denote the common kernel of both $d_{P} f$ and $\chi(\Lambda, x)$. By generic smoothness $d_{P} \bar{f}$ is surjective, hence

$$
\operatorname{dim} \operatorname{ker} d_{P} \bar{f}=(n-1)-(n-c)=c-1 .
$$


But ker $d_{P} \bar{f}=K_{P} \cap T_{P} \bar{\Phi}$, where $T_{P} \bar{\Phi}$ has dimension $n-1$, so either $K_{P} \subset$ $T_{P} \bar{\Phi}$, the two kernels coincide and $\operatorname{dim} K_{P}=c-1$, or they are different and $\operatorname{dim} K_{P}=c$. Since the structure of scheme on $\Phi$ is given by the minors of the characteristic map, this proves that $\mu(P, \Phi)$ is at least $c-1$ in the former case, and at least $c$ in the latter.

Example 1. The multiplicity $\mu\left(x, F_{\Lambda}\right)$ can be strictly greater than $c$. We see now an example of such a situation, in which the fibres of the Gauss map have special properties of tangency to the focal locus.

Let us consider a birational map $\varphi: F \rightarrow S$ between two surfaces $F, S \subset$ $\mathbb{P}^{6}$. Then

$$
\begin{array}{rlcc}
\psi: & F & \rightarrow & \mathbb{G}(3,6) \\
x & \mapsto & \left\langle\mathbb{T}_{x} F, \varphi(x)\right\rangle
\end{array}
$$

defines a rational map on $F$. We define $X$ as the closure of the variety swept by the 3-planes in $\mathbb{P}^{6}$ belonging to the image of $\psi$. If the choice of $F, S$ and $\varphi$ is general, then $X$ is a variety of dimension 5 whose Gauss image has dimension 4 , and its focal locus is $F$, with codimension 3 . If $x$ is a general point of $F$, then any line passing through $x$ and contained in $\left\langle\mathbb{T}_{x} F, \varphi(x)\right\rangle$ is a fibre of the Gauss map.

Let $P=(\Lambda, x)$ project to a general point of $F$. By direct computation, $x$ results to be the only focus on $\Lambda$, with multiplicity $4=c+1$.

It is nonetheless true that $\operatorname{dim} K_{P}=2=c-1$. The kernels of $d_{P} \bar{f}$ and $d_{P} f$ indeed coincide and are generated by the directions of the curves in $\mathcal{I}$ of the form $(\Lambda(t), x)$, where $\Lambda(t)$ varies in the star of lines of centre $x$ contained in $\psi(x)$.

The just constructed example belongs to the class of hyperbands of [AGL]. It can be generalized to a whole series of analogous examples with larger dimensions and increasing difference between the multiplicity of the focal locus and the codimension $c$.

Example 2. The Severi varieties.

Let $F$ denote one of the four Severi varieties (see the Introduction). Let $X=S F$ be its secant variety, i.e., the closure of the union of lines joining two distinct points of $F$. There are several known facts about $F$.

Proposition 2.4 ([Z2]). Let $F \subset \mathbb{P}^{N}$ be a Severi variety. Then the following hold:

- $F$ has dimension $m \in\{2,4,8,16\}$;

- $F$ is embedded in a projective space of dimension $N=3 m / 2+2$;

- the secant variety $X$ of $F$ is a normal cubic hypersurface in $\mathbb{P}^{N}$;

- $X$ is isomorphic to the dual variety of $F$;

- the singular locus of $X$ coincides with $F$.

As $X$ is a hypersurface, its Gauss image is the same as its dual variety, which is $F$. Then $X$ has Gauss rank $m$ and the fibres of the Gauss map 
$\gamma$ of $X$ are linear subspaces of dimension $m / 2+1$. As shown in [Z2], the intersection with $F$ of the secant lines of $F$ passing through a general point $x \in X$ is a quadric generating a space of dimension $m / 2+1$. This space is precisely the fibre of the Gauss map passing through $x$, and $B$ is just the family of such spaces. As the quadric depends on the fibre $\Lambda$ to which $x$ belongs and not on $x$, we will denote it by $Q_{\Lambda}$.

The degree of the focal scheme on $\Lambda$ must equal the Gauss rank, so it is $m$, i.e., 2, 4, 8 or 16 respectively. But the focal locus of $B$ on $\Lambda$ has to be $Q_{\Lambda}$, of degree 2 , because it has to be contained in the singular locus of $X$. This shows that $F_{\Lambda}$ coincides with $Q_{\Lambda}$ set-theoretically, but, as a scheme, appears with multiplicity $1,2,4,8$ respectively in the four cases. This fact is confirmed by Proposition 2.2, because in this case $c=m / 2+1$ and the multiplicity $\mu(x, \bar{F})$ is equal to $m / 2=c-1$.

\section{Gaps on the codimension and uniqueness of Severi examples.}

The results of the previous section allow us to state some bounds on the possible codimension of the focal locus for the family of the fibres of the Gauss map and some uniqueness results.

Theorem 3.1. Let $X$ be a variety of dimension $n$ with degenerate Gauss image $\gamma(X)$ of dimension $r$ with $r \geq 2$. Let $c$ denote the codimension of the focal locus $F$ in $X$. Then:

(i) $c \leq r+1$;

(ii) if $c=r+1$, then $X$ is a cone of vertex a space of dimension $(n-r-1)$ over a variety of dimension $r$;

(iii) if $c \leq r$ and the reduced focal hypersurfaces are not linear, then $c \leq$ $r / 2+1$

(iv) if $c=r / 2+1$, then either the multiplicity of the focal hypersurfaces in the fibres of $\gamma$ is at least $c$ and they are set-theoretically linear, or the multiplicity is $r / 2$ and the reduced focal hypersurfaces are quadrics.

Proof. (i) Let $F_{\Lambda}$ be the focal scheme on a general fibre $\Lambda$ of the Gauss map. Then $F_{\Lambda}$ is a hypersurface of degree $r$, and from Corollary 2.3, it has multiplicity $\mu\left(x, F_{\Lambda}\right) \geq c-1$ at a general point $x$. Hence $c-1 \leq \mu\left(x, F_{\Lambda}\right) \leq r$, which implies (i).

(ii) If $c=r+1$, then a general $F_{\Lambda}$ is a hyperplane in $\Lambda$ with multiplicity $r$. On the other hand $\operatorname{dim} F=n-r+1=\operatorname{dim} F_{\Lambda}$, so $F_{\Lambda}$ is a fixed $(n-r+1)$ plane and $X$ is a cone over it.

(iii) and (iv) Assume $r / 2+1 \leq c \leq r$ : Then $\mu\left(x, F_{\Lambda}\right) \geq c-1 \geq r / 2$. So the degree of the reduced focal locus on $\Lambda$ is $r / \mu\left(x, F_{\Lambda}\right) \leq 2$. If $\mu\left(x, F_{\Lambda}\right) \geq$ $c$, then the hypothesis implies that $\mu\left(x, F_{\Lambda}\right)=r$, so $\left(F_{\Lambda}\right)_{\text {red }}$ is linear. If $\mu\left(x, F_{\Lambda}\right)=c-1$, then our hypothesis implies $c=r / 2+1$ and $\mu\left(x, F_{\Lambda}\right)=$ $r / 2$. 
Remark 3.2. The description of varieties with Gauss rank $r=1$ is classical (see $[\mathbf{F P}]$ for a modern account), the description of varieties with $r=2$ has been accomplished recently by Piontkowski $([\mathbf{P}])$. His uniqueness theorem for the case in which the focal locus has codimension 2 can be extended as follows:

Theorem 3.3. Let $X$ be a variety of dimension $n$ with degenerate Gauss image $\gamma(X)$ of dimension $r \geq 2$ and let $F$ be the focal locus of the family of fibres of $\gamma$. Assume that the codimension of $F$ in $X$ is $c=r / 2+1$ and that the focal hypersurfaces are quadrics counted with multiplicity $r / 2$.

(i) If $F$ is irreducible, then $X$ coincides with $S F$, the secant variety of $F$, and $n \geq 3 r / 2+1$

(ii) if moreover $n=3 r / 2+1$ and a general quadric $F_{\Lambda}$ is smooth, then $F$ is a Severi variety;

(iii) if $n \geq 3 r / 2+1$, then the rank of the quadrics $F_{\Lambda}$ is at most $r / 2+1$, and, if equality generically holds, then $F$ is (a cone over) a Severi variety of dimension $r$.

Proof. (i) Since the focal hypersurfaces have set-theoretically degree 2, then the lines which are contained in the fibres of $\gamma$ are all secant lines of $F$. Hence $X \subset S F$. On the other hand, the family of lines obtained in this way has dimension $r+\operatorname{dim} \mathbb{G}(1, n-r)$, which is equal to $2 \operatorname{dim} F$. This proves that $X=S F$. A standard count of parameters shows that the family of quadrics passing through a general point of $F$ has dimension $r / 2$ and that the intersection of two focal quadrics is always nonempty.

Let us denote by $B$ as usual the family of fibres of $\gamma$. Let $\Sigma \subset B \times$ $B \times F$ be the set of triples $(\Lambda, \mathrm{M}, P)$ such that $P \in F_{\Lambda} \cap F_{\mathrm{M}}$. Considering the projections from $\Sigma$ to $B \times B$ and to $F$, one gets easily that a general intersection $F_{\Lambda} \cap F_{\mathrm{M}}$ has dimension $n-3 r / 2-1$. This proves (i).

(ii) We can assume without loss of generality that $X$ is nondegenerate (we can always restrict to the projective subspace of minimal dimension in which $X$ is contained). Suppose first that $X$ is a hypersurface. We have that $F$ is a variety of dimension $r$ covered by a $r / 2$-dimensional family of nonsingular quadrics of dimension $r / 2$, such that a general pair of such quadrics meet at a point. This property of the family of quadrics allows us to argue as in [Z2, Lemma 5] and deduce, as is done there, that $F$ is smooth. Therefore $F$ is a Severi variety by definition.

Suppose now that $X \subset \mathbb{P}^{N}, N=3 r / 2+2+k, k>0$. The image of $X$ in a projection with centre a general $(k-1)$-plane of $\mathbb{P}^{N}$ is a hypersurface, which is the secant variety of a Severi variety $F^{\prime}$ by the previous argument. Since the focal locus of $X$ projects to $F^{\prime}$, we have that the general section of $F$ by a linear subspace of dimension $3 r / 2+2$ is a Severi variety. By Corollary 1 of $[\mathbf{Z 4}]$ Severi varieties are unextendable, hence $F$ must be a cone over $F^{\prime}$, which is impossible because in that case the quadrics $F_{\Lambda}$ would be singular. 
(iii) Assume that the rank of $F_{\Lambda}$ is $\geq r / 2+1$. Let $h:=(n-r / 2-1)-(r+1)=$ $n-3 r / 2-2, h \geq-1$. Cutting $F$ with $L$, the intersection of $h+1$ general hyperplanes, we get $F^{\prime}:=F \cap L$, of dimension $r$, containing a $r$-dimensional family of quadrics of dimension $r / 2$ and generically of maximal rank $r / 2+1$. Moreover the variety of secant lines of $F^{\prime}$ coincides with $S F \cap L$, so it has dimension $3 r / 2+1$. By (ii) we obtain that $F^{\prime}$ is a Severi variety. Again, since Severi varieties are unextendable, we can conclude that $F$ is a cone over $F^{\prime}$ with vertex a linear space of dimension $h$. In particular, the rank of $F_{\Lambda}$ is $r / 2+1$.

Remark 3.4. Smooth varieties $F$ such that $X=S F$ satisfies the assumptions of Theorem 3.3 are precisely the varieties studied by Ohno in [O].

\section{Examples of focal hypersurfaces of any degree or with arbitrarily high multiplicity.}

In this section we give two series of examples: The former show that the focal hypersurfaces in the fibres of the Gauss map can appear with arbitrarily high multiplicity, the latter have focal hypersurfaces of arbitrary degree. Note that in Example 3 the variety $X$ is not a hypersurface if $m \geq 3$.

\section{Example 3. Secant varieties of Scorza varieties.}

Let $F$ be one of the following varieties: $v_{2}\left(\mathbb{P}^{m}\right), \mathbb{P}^{m} \times \mathbb{P}^{m}, \mathbb{G}(1,2 m+1)$. They are contained in the projective spaces of dimension $\left(\begin{array}{c}m+2 \\ 2\end{array}\right)-1,(m+$ $1)^{2}-1,\left(\begin{array}{c}2 m+2 \\ 2\end{array}\right)-1$ respectively, and have dimensions $m, 2 m, 4 m$. In all cases $F$ is defined by suitable minors of a matrix of variables, and precisely by $2 \times 2$ minors of a symmetric $(m+1) \times(m+1)$ matrix in the first case, by $2 \times 2$ minors of a generic $(m+1) \times(m+1)$ matrix in the second case, by Pfaffians of $4 \times 4$ minors of a skew-symmetric matrix of order $2 m+2$ in the third one. These varieties are considered by Zak in $[\mathbf{Z 3}]$, and named by him Scorza varieties.

Let $X$ be the secant variety of $F$ : It is defined by minors of the same matrix as $F$ of order one more. Then the fibres of the Gauss map of $X$ have respectively dimension $2,3,5$, the focal hypersurfaces are quadrics of dimensions 1, 2, 4, which appear with multiplicity $m-1,2 m-2,4 m-4$ respectively.

\section{Example 4. Higher secant varieties of Scorza varieties.}

Let $X=S^{m-1} F$ be the variety of $m$-secant $(m-1)$-planes of $F$, where $F$ is one of the varieties appearing in the previous example. $X$ is the maximal proper secant variety of $F$ and it is the hypersurface defined by the determinant (or the Pfaffian) of the matrix considered in previous example. The varieties $X$ and $F$ result to be mutually dual, so $r=m, 2 m, 4 m$ respectively. The focal locus of the Gauss map is $S^{m-2} F$. The focal hypersurfaces are the maximal secant varieties of $v_{2}\left(\mathbb{P}^{m-1}\right), \mathbb{P}^{m-1} \times \mathbb{P}^{m-1}, \mathbb{G}(1,2 m-1)$ 
respectively. They have degree $m$ and appear with multiplicity respectively $1,2,4$.

Remark 4.1. The class of Scorza varieties includes also $\mathbb{P}^{m} \times \mathbb{P}^{m-1}$ and $\mathbb{G}(1,2 m)$, for all $m \geq 3$. Also their secant varieties have properties similar to above. They are defined by minors of matrices of type $(m+1) \times(m+2)$ and of skew-symmetric square matrices of type $2 m+1$ respectively.

Acknowledgment. We would like to thank Prof. Fyodor Zak for some useful suggestions and the referees for interesting remarks and criticism.

\section{References}

[AG] M.A. Akivis and V.V. Goldberg, On the structure of submanifolds with degenerate Gauss maps, Geom. Dedicata, 86 (2001), 205-226, MR 2003a:53014.

[AGL] M.A. Akivis, V.V. Goldberg and J.M. Landsberg, Varieties with degenerate Gauss mapping. Preprint, 2001, math.AG/9908079.

[FP] G. Fischer and J. Piontkowski, Ruled Surfaces, Viehweg, 2001, MR 2003f:14044, Zbl 0976.14025.

[GH] Ph. Griffiths and J. Harris, Algebraic geometry and local differential geometry, Ann. Sci. École Norm. Sup. (4), 12 (1979), 355-452, MR 81k:53004, Zbl 0426.14019.

[K] S.L. Kleiman, Concerning the Dual Variety, Progr. Math., 11, Birkhäuser, Boston, 1981, 386-396, MR 82m:14033, Zbl 0468.14018.

[L1] J.M. Landsberg, On degenerate secant and tangential varieties and local differential geometry, Duke Math. J., 85(3) (1996), 605-634, MR 98d:14066, Zbl 0879.14025.

[L2] , Algebraic Geometry and Projective Differential Geometry, Lecture Note Series, 45, Seoul National Univ., Seoul, Korea, 1999, MR 2001i:14069, Zbl 1012.14015.

[MT] E. Mezzetti and O. Tommasi, On projective varieties of dimension $n+k$ covered by k-spaces, Illinois J. Math., 46(2) (2002), 443-465, MR 2003k:14067.

[O] M. Ohno, On degenerate secant varieties whose Gauss maps have the largest images, Pacific J. Math., 187(1) (1999), 151-175, MR 2000f:14084, Zbl 0939.14029.

[P] J. Piontkowski, Developable varieties of Gauss rank 2, Internat. J. Math., 13(1) (2002), 93-110, MR 2003g:14078.

[S] C. Segre, Preliminari di una teoria delle varietà luoghi di spazi, Rend. Circ. Mat. Palermo (1), 30 (1910), 87-121, also in Opere Scelte, Vol. 2, ed. Cremonesi, Roma, 1958.

[Z1] F.L. Zak, Projections of algebraic varieties, Math. USSR Sbornik, 44 (1983), 535544, MR 84i:14012, Zbl 0511.14026.

[Z2] _ Severi varieties, Math. USSR Sbornik, 54 (1986), 113-127, MR 86k:14029, Zbl 0592.14036.

[Z3] - Tangents and Secants of Algebraic Varieties, Translations of Mathematical Monographs, 127, Providence, RI, American Mathematical Society, 1993, MR 94i:14053, Zbl 0795.14018. 
[Z4] Some properties of dual varieties and their applications in projective geometry, Lecture Notes in Math., 1479, Springer, Berlin, 1991, 273-280, MR 94a:14041, Zbl 0793.14026.

Received October 24, 2002 and revised February 11, 2003. Work supported by funds of the University of Trieste (fondi 60\%) and MIUR, project "Geometria sulle varietà algebriche". The first Author acknowledges support from the Newton Institute of Cambridge, during part of the preparation of this paper.

Dipartimento di Scienze Matematiche

UNIVERSitÀ Di TRIESTE

Via Valerio 12/1, 34127 Trieste

ITALY

E-mail address: mezzette@univ.trieste.it

Department of Mathematics

UNIVERSiTy OF NiJMEGEN

Toernooiveld, 6525 ED NiJMegen

The Netherlands

E-mail address: tommasi@math.kun.nl 